\title{
Comorbidity of Alcohol Use Disorder and Depression Among Patients Attending a Tertiary Hospital in the Niger Delta Region of Nigeria
}

\author{
Okeafor Chukwuma Ugochukwu, Chukwujekwu Chidozie Donald*, \\ Stanley Princewill Chukwuemeka \\ Department of Neuropsychiatry, University of Port Harcourt Teaching Hospital, Port Harcourt, Rivers State, Nigeria \\ Email address: \\ chidozie.chukwujekwu@uniport.edu.ng (C. C. Donald) \\ ${ }^{*}$ Corresponding author
}

\section{To cite this article:}

Okeafor Chukwuma Ugochukwu, Chukwujekwu Chidozie Donald, Stanley Princewill Chukwuemeka. Comorbidity of Alcohol Use Disorder and Depression Among Patients Attending a Tertiary Hospital in the Niger Delta Region of Nigeria. American Journal of Psychiatry and Neuroscience. Vol. 4, No. 3, 2016, pp. 38-42. doi: 10.11648/j.ajpn.20160403.11

Received: April 30,2016; Accepted: May 9, 2016; Published: May 17, 2016

\begin{abstract}
The study aims to ascertain the co-morbidity of Alcohol and Depression among patients attending the general outpatient clinic of the University of Port Harcourt Teaching Hospital. Via a system sampling method; 470 consenting subjects were enlisted in the study. The Alcohol Use Disorders Identification Inventory (AUDIT) and the Beck's Depression Inventory (BDI) were administered to each. Only one hundred and eighty five subjects met the criteria for the second stage of the study, (a score of 18 and above on the BDI and/or a score of 5 and above on AUDIT). Results showed that there was a significantly higher prevalence of Alcohol Use Disorders among those with higher depressive symptoms. Furthermore, about (45.8\%) half of these with Alcohol Use Disorders were diagnosed with depression in comparison to $26.0 \%$ of those without Alcohol Use Disorder. In agreement with other studies, there was a greater likelihood of depression among the subjects who were diagnosed with AUD. Nevertheless, the specific mechanism underlying this co-morbidity is yet to be elucidated.
\end{abstract}

Keywords: Depression, Alcohol, Co-morbidity, Disorder

\section{Introduction}

Co-morbidity has been referred to as one of the most important challenges in the field of mental health [1]. When two disorders or illnesses occur in the same person simultaneously or sequentially they are described as comorbid. Majority of individuals who meet the criteria for a major psychiatric illness will also meet diagnostic criteria for at least one additional disorder [2]. Co-morbid conditions follow a more chronic and treatment resistant course than those with only a single disorder and they are more likely to experience severe psychosocial impairment [1, 3, 4]. These concerns are of particular importance to Depression and Substance Use Disorders, both of which are highly prevalent in the general population and widely implicated to co-morbid relationships across the life span $[5,6,7]$.

Although some studies have demonstrated the role of alcohol abuse in psychopathology [8], and many works have been done in the Western world in the relationship between AUD and Depression $[9,10,11]$, scarcely has any work been done in the area of association between AUD and Depression in Nigeria. Adewuya 2006 [12] in a study of prevalence of major depressive disorder in college students with alcohol related problems found depressive rates of $17.2 \%$ and $23.8 \%$ among alcohol abusers and those with alcohol dependence respectively. This study, though multi-centered, was conducted in six post-secondary institutions in a particular state of Western Nigeria. Therefore it does not reflect the diverse socio-cultural background of the different parts of Nigeria.

There is no better place for this study to be done than in the Niger Delta Area of Nigeria. Rivers state serves as the unofficial centre of Nigeria's booming oil industry and has been awash with violence, militancy, kidnapping and other violent crimes for almost two decades now. Most of these 
acts have been associated with substance and alcohol abuse [13]. Understanding the nature of the co-morbid relationship between Alcohol Use Disorders and Depression may have important implications for improving nosology as well as for developing better models of preventing these disorders.

\section{Materials and Methods}

This prospective cross-sectional study was conducted at the general outpatient clinic of the University of Port Harcourt Teaching Hospital (UPTH) over a six month period, from February 2011 - July 2011.

\subsection{Instruments}

For this study, the instruments employed are as follows:

1 Alcohol Use Disorders Identification Test (AUDIT)

2 Beck's Depression Inventory (BDI)

3 Composite International Diagnostic Interview (CIDI)

4 Socio-demographic protocol designed by the authors

The Alcohol Use Disorder Identification Test (AUDIT) is a self rated 10-item questionnaire with each item scored 0-4, giving a total score of 40 . Some studies have shown its validity and reliability in the detection of hazardous drinking, alcohol abuse and dependence. It has been reported that a score of 5 provides a good tradeoff between sensitivity and specificity $[15,16,17]$. It has been revalidated and used in Western Nigeria [18].

The Beck's Depression Inventory (BDI) is a 21 item self report inventory [19]. It is one of the most used instruments for screening and estimating the intensity of Depression. It has been revised in the second edition, to reflect the Diagnostic and Statistical Manual (DSM-IV) diagnostic criteria [20]. In terms of its psychometric properties, the second edition of BDI has been positively correlated with the Hamilton Depression Rating Scale (HDRS) with a Pearson Coefficient $(\delta)$ of 0.71 , showing good agreement. The test was also shown to have high test-retest reliability $(\delta=0.93)$ and a high internal consistency $(\delta=0.91)$ [21]. Each item has four statements and the patient chooses that which applies best to their feelings over the previous two weeks. A value of $0-3$ is assigned to each answer and then the total is computed to determine the severity of Depression. The scores range from 0 to $63[19,22]$. The questionnaire can be completed in 5 minutes. It has been revalidated and used in Western Nigeria and a score of 18 and above has been shown to be indicative of Depressive Disorder [23, 24].

The Composite International Diagnostic Interview (CIDI) is used for the assessment of mental disorders and to provide diagnosis according to the International classification of Disease $10^{\text {th }}$ Revision (ICD-10) of the World Health Organization (1992) and the fourth edition of Diagnostic and Statistical Manual of mental disorders (DSM-IV) of the American Psychiatric Association (1994). It is particularly useful in epidemiological studies of mental disorders and can be completed in a relatively short time. The inter-rater reliability has been found to be excellent, the test-retest reliability is good and the validity has been demonstrated to be good [25]. It has been widely used in different studies within Nigeria [23, 24, 26].

The paper and penal form of the world Mental Health CDI version 3.0 was used for this study. For the purpose of this study and due to the unavailability of the diagnostic algorithm of CIDI, diagnosis of Alcohol Used Disorders and major Depression were made by matching the symptoms generated by CIDI with the DSM-IV diagnostic criteria.

\subsection{Procedure}

The study took place in 2 phases; in the first phase, the AUDIT and BDI instruments were administered to 470 subjects. Diagnosis of major depression was made according to the DSM-IV diagnostic criteria using the Composite International Diagnostic Criteria (CIDI). One hundred and eighty five (185) subjects met the criteria for the second stage. Subjects who qualified for this stage had a score of 18 and above on the BDI and/or a score of 5 and above on AUDIT.

Systematic sampling technique was used to select the subjects. Every fourth eligible patient registered at the general outpatient clinic for the day was selected from the medical records register. Ballot method was used to select the first patient for the day from the first four eligible patients registered for a particular session. Subsequently, every fourth from the position selected by ballot was selected until the end of the clinic session. Consenting patients were recruited as described above on each clinic day until the required sample size was achieved.

Before the commencement of this study, approval of the ethical committee of the University of Port Harcourt Teaching Hospital (UPTH) was sought and informed consent obtained from the subjects to be involved in the research. The data was analyzed using the Statistical Package for Social Sciences (SPSS) at 5\% level of significance and $95 \%$ confidence interval.

Proportions or categorical parameters were analyzed with chi-square test or 2-tailed Fisher's exact test as appropriate. Multivariate correlation analysis was used to establish measures of association between socio-demographic variables and Alcohol use Disorders, Depression and comorbidity. Continuous variables like the Beck's Depression Inventory (BDI) scores were compared by the student's t-test.

\section{Results}

Fifty nine $(12.6 \%)$ of the subjects were diagnosed with Alcohol Use Disorders according to the DSM-IV criteria. One hundred and thirty four $(28.5 \%)$ of the subjects were diagnosed with a Major Depressive Episode and twenty seven $(5.7 \%)$ of the subjects were diagnosed with both Alcohol Use Disorders and Major Depressive Episode. The rate of Depression among those with AUD was found to be $45.8 \%$.

Correlation of the BDI scores against the AUDIT scores with Pearson's correlation was significantly positive with a Pearson's coefficient $(\delta)$ of $0.5 \%$. In the whole study 
population, the mean Beck's Depression Inventory (BDI) score was $12.67 \pm 9.43$, while the mean Alcohol Use Disorder Identification Test (AUDIT) score was $2.36 \pm 4.72$. The Student t-test done for comparison of the mean BDI scores between those without Alcohol Use Disorders (11.85 \pm 9.21) and those with Alcohol Use Disorders (18.34 \pm 9.02$)$ revealed a significant difference $(\mathrm{t}=-5.15, \mathrm{p}=0.100)$. There was also a significant difference $(t=-2.728, p=0.007)$ between the mean AUDIT scores of those with Depression $(3.46 \pm 6.00)$ and those without Depression (1.92 \pm 4.02$)$.

Table 1 shows that there was a significantly higher prevalence of Alcohol Use Disorders among the respondents with higher depressive symptoms rating. The prevalence of AUD among the subjects that scored 18 and above on BDI was $18.7 \%$ while the prevalence of AUD among the subjects that scored less than 18 on BDI was $9.7 \%\left(X^{2}=7.500, d f=1, \mathrm{p}\right.$ value $=0.006, \mathrm{OR}=2.14,95 \%$ C. I, 1.19 to 3.85$)$.

In table 2, nearly half (45.8\%) of those with Alcohol Use Disorders were also diagnosed with Depression in comparison to $26.0 \%$ of those without Alcohol Use Disorders. This was statistically significant $\left(X^{2}=9.852, d f=\right.$ 1 , p value $=0.002$, OR $2.40,95 \%$ C. I, 1.32 to 4.34$)$.

Table 1. Association between depression rating and alcohol use disorders.

\begin{tabular}{|c|c|c|c|c|c|c|}
\hline Depression Rating Category & Without AUD n (\%) & With AUD n (\%) & Total & $X^{2}$ & $d f$ & Pvalue \\
\hline Minimal to mild (BDI scores $<18$ ) & $289(90.3)$ & $31(9.7)$ & 320 & \multirow[t]{3}{*}{7.500} & \multirow[t]{3}{*}{1} & \multirow[t]{3}{*}{0.006} \\
\hline Moderate to severe (BDI score $\geq 18$ ) & $122(81.3)$ & $28(18.7)$ & 150 & & & \\
\hline Total & 411 & 59 & 470 & & & \\
\hline
\end{tabular}

Table 2. Association between alcohol use disorders and depression.

\begin{tabular}{|c|c|c|c|c|c|c|}
\hline & Without Depression n (\%) & With Depression n (\%) & Total & $X^{2}$ & $d f$ & P value \\
\hline Without AUD & $304(74.0)$ & $107(26.0)$ & 411 & 9.852 & 1 & 0.002 \\
\hline With AUD & $32(54.2)$ & $27(45.8)$ & 59 & & & \\
\hline Total & 336 & 134 & 470 & & & \\
\hline
\end{tabular}

\section{Discussion}

In agreement with other studies $[14,15,16]$ there was a greater likelihood of depression among the subjects that was diagnosed with AUD. The 45.8\% Depression rate among those with AUD in this study is obviously higher than the $11.3 \%$ and $27.9 \%$ reported respectively for Alcohol abuse and Alcohol dependence by Adewuya among college students with alcohol related problems [12]. The higher value is not surprising since this study was carried out among a clinic sample. Also the socio-cultural differences may also account for this higher rate.

The significantly higher depressive symptom rating among the subjects with AUD agrees with other reports [17, 18]. This and the fact that there was a significantly higher mean BDI score among those with AUD in comparison to those without AUD further strengthens the relationship between AUD and Depression.

Moreover, Leonardson et al [19] had earlier demonstrated a significant positive correlation between BDI scores and Alcohol Use Disorders Identification Test (AUDIT) scores amongst American Indians. This significant correlation was replicated in this study.

Although the co-morbidity of AUD with Depression has been widely studied in both clinical and epidemiological investigations, the specific mechanisms of these associations remain a source of debate. Indeed, 'drinking to forget' andother adages reflect common awareness in society that Alcohol consumption may be causally linked to Depression or other psychological difficulties. One widely accepted hypothesis is that this form of co-morbidity may reflect a causal relationship of Alcoholism and Depression. Support for a causal association is based partly on the observation that alcohol is commonly used to self medicate symptoms of negative effect and that AUD develops as a secondary diagnosis to Depression [5, 20]. Moreover the evidence for a causal relationship is not unidirectional as Alcoholism is often observed as a primary disorder, and the presence of problem drinking itself may generate depressive syndrome [21]. The cross sectional nature of this study could not allow for temporal relationships to be examined. Hence longitudinal studies with focus on temporal relationships between AUD and Depression would help to elucidate this relationship.

An alternative explanation of co-morbidity also gained support by positing that shared aetiologic factors contribute to the manifestations of each of these disorders, or that Alcoholism and Depression represent different stages of the same disease entity [14]. Similar to the causal hypothesis, there is also inconsistent evidence for the shared etiology model [22]. Thus despite the strong association of Alcoholism with Depressive Disorders, no universal consensus has been reached regarding the specific mechanisms underlying these associations [27, 29].

It is therefore possible that within the population of individuals experiencing Depression and Alcohol use comorbidity, multiple pathways of association may be acting simultaneously. The combination of shared diathesis and causal models of association (in both directions) may therefore explain numerous discrepancies noted in the literature, and explain why the association of Depression with drug or Alcohol dependence does not show the unidirectional patterns common to other forms of co-morbidity. 


\section{Conclusion}

The result of this study clearly shows that there is a significantly higher prevalence of depression among patients with AUD and vice versa. Therefore those suffering from AUD are more vulnerable to suffer depression, while those diagnosed with major depression are more likely to use alcohol to ameliorate their depressive symptoms and hence more vulnerable to suffer AUD. Consequently, it is highly recommended that in the clinical assessment of AUD patients, depressive symptoms should be diligently explored; the reverse is equally correct. High index of suspicion of this co-morbidity, on the part of clinicians, comprehensive clinical assessment of these patients and prompt institution of treatment will translate to more effective management of sufferers.

\section{Limitation}

This study was cross-sectional in nature. This made it difficult to ascertain the temporal relationship between Alcohol Use Disorders and Depression among the subjects with these disorders.

\section{References}

[1] Kendall P, Clarkin J. Introduction to special section: Comorbidity and treatment implications. Journ Consul Clin Psychology. 1992; 60: 833-834.

[2] Kessler R, McGinagle K, Zhao S, Nelson C, Hughes M, Eshleman S. et al (1994). Lifetime and 12-month prevalence of DSM-III R Psychotic disorders in the United States: Results from the National Co-morbidity Survey. Arch of Gen Psychiatry; 51, 8-19.

[3] Hagnell, O \& Grasbeck, A. Co-morbidity of anxiety and depression in the lundby 25 -year prospective study: the pattern of subsequent episodes. In: Maser, J. \& Cloninger, C. (Eds). Co-morbidity of mood and anxiety disorders. Washington, DC: American Psychiatric Press Inc. 1990; pp. 139-152.

[4] Hirschfeld R., Hasin D., Keller M., Endicort J. and Wunder J. Depression and alcoholism: Co-morbidity in a longitudinal study. In: Maser J and Cloninger C. (Eds). Washington, DC: American Psychiatric Press Inc, 1990; pp. 293-304.

[5] Hasin D, Endicott J, Keller M B. Alcohol problems in psychiatric patients: 5-year course. Compr Psychiatry 1991; 32 (4): 303-316.

[6] Meyer R, Kranzler H. Alcohol abuse/dependence and comorbid anxiety and depression. In: Maser J, Cloninger C (Eds). Co-morbidity of Mood and Anxiety disorders. American Psychiatric Press, Washington, DC; 1990: pp. 283292.

[7] Rhode P, Lewinson P M., \& Seeley, JR. Co-morbidity of unipolar depression: Co-morbidity with other mental disorders in adolescents and adults. JournAbn Psychology 1991; 100 (2), 214-222.

[8] Obot I S, Olaniyi A. Drug related psychotic disorders in four Nigerian hospitals. NigJ Psychiatry 1990; 8, 13-16.
[9] Gilman S E, Abraham H D. A longitudinal study of the order of onset of alcohol dependence and major depression. Drug Alcohol Depend 2001; 63 (3), 277-286.

[10] Wang J, Pattern S B. Alcohol consumption and major depression: Findings from a follow up study. Can J Psychiatry 2001; 46 (7): 632-638.

[11] Bosch I, VandenBrinck W, Pennins B W, Wall M, M, Hasin D. Alcohol Use Disorder severity predicts first incidence of depressive disorders. Psychol Medicine 2011; 26: 1-9.

[12] Adewuya A O. Prevalence of major depressive disorder in Nigerian college students with alcohol-related problems. Gen Hosp Psychiatry 2006; 28 (2): 169-173.

[13] Brisbe S, Ordinioha B, Dienye P O. (2011). Intersection between alcohol abuse and intimate partner's violence in rural Ijaw Community in Bayelsa State, South-South Nigeria. J Interpers Violence 2012; 27 (3): 513-522.

[14] Swedsen J D, Merikangas K R, Canino G I, Kessler R. C, Rubio-Stipec M, Angst J (1988). The co-morbidity of Alcoholism with Anxiety and Depressive Disorders in four geographic communities. Compr Psychiatry 1998; 39: 176-184.

[15] Graham K, Massak A, Demers A, Rehm J. Does the association between alcohol consumption and depression depend on how they are measured? Alcoholism: Clin and Exp Research 2007; 31 (1): 78-88.

[16] Hasin D S, Grant B F. Major Depression in 6050 former drinkers: Associations with past alcohol dependence. Arch Gen Psychiatry 2002; 59: 794-800.

[17] Kenneth R C, Martin P, Stephanie A G. Meta-analysis of depression and substance used among individual with use disorders. J Subs Abuse Treat 2009; 37 (2), 127-137.

[18] Leonardson G R, Kemper E., Ness F K., Koplin B A, Daniels M C, Leonardson G. A. Validity and reliability of the AUDIT and CAGE-AID in Northern plains American Indians. Psycho Rep 2005; 97 (1): 161-166.

[19] Mendelson J, Mello N. Medical progress: Biologic concomitants of alcoholism, New English Journ of Med 1979; 301: 912-921.

[20] Shuckit M. A., Tipp J. E., Bergman M., Reich W., Hasselbrook U. M. \& Smith T. L. (1997). Comparison of induced and independent major depressive disorders in 2, 945 alcoholics. Am J Psychiatry 1997; 154 (7): 948-957.

[21] Beck AT, Steer RA, Carbin M. G. Psychometric properties of the Beck Depression Inventory: twenty five years of evaluation. ClinPsychol Rev 1988; 8: 77-100.

[22] Blacker D. Psychiatric rating scales. In: Sadock BJ, Sadock VA, Ruiz P. Kaplan and Sadock's comprehensive textbook of Psychiatry. Ninth Edition. Philadelphia, PA: Lipincot, Williams and Wilkins, 2009; 1032-1059.

[23] Adewuya AO. Validation of the Alcohol Use Disorder Identification Test (AUDIT) as a screening tool for alcohol related problems among Nigerian university students. Alcohol and Alcoholism 2005; 40: 575-577.

[24] Gureje O, Uwakwe R., Oladeji B., Makanjuola UD, Esan O. Depression in adult Nigerians; results from the Nigerian Survey of Mental Health and Well Being. J Affect Disord 2010; 120: 158-164. 
[25] Andrews G, Peters L. The psychometric properties of the Composite International Diagnostic Interview. Soc Psychiatry Psychiatr Epidemiol 1998; 33: 80-88.

[26] Awaritefe A. The Beck Depression Inventory in relation to some commonly used tests in Nigeria. Nigerian Journal of Basic and Applied Psychology 1998; 1: 23-28.

[27] Schuckit M A, Smith T L, Kalmijin K. Relationships among independent major depressive, alcohol use and other substance use and related problems over 30 years in 397 families. Journ Stud Alc Drugs 2013; 74: 271-279.

[28] Magidson J F, Saal W, Nel A, Remmert E, Kagee A. Relationship between depressive symptoms, alcohol use and anti-retroviral therapy adherence among HIV- infectad, clinic attending patients in South Africa. J Health Psychol Feb. 15 20161359105316628743. 